\title{
Consanguinity Protecting Effect Against Breast Cancer among Tunisian Women: Analysis of BRCA1 Haplotypes
}

\author{
Imen Medimegh ${ }^{1}$, Wafa Troudi ${ }^{1 *}$, Ines Omrane ${ }^{1 \&}$, Hajer Ayari ${ }^{1 \&}$, Nancy \\ Uhrhummer $^{3}$, Hamdi Majoul' ${ }^{1}$, Farhat Benayed ${ }^{2}$, Amel Mezlini², Yves-Jean \\ Bignon $^{3}$, Catherine Sibille ${ }^{4}$, Amel Benammar Elgaaied ${ }^{1}$
}

\begin{abstract}
The purpose of this study is to assess the effect of consanguinity on breast cancer incidence in Tunisia. We conducted a case-control study to evaluate the involvement of heterozygote and homozygote haplotypes of BRCA1 gene SNPs according to consanguinity among 40 cases of familial breast cancer, 46 cases with sporadic breast cancer and 34 healthy controls. We showed significant difference in consanguinity rate between breast cancer patients versus healthy controls $P=0.001$. Distribution of homozygous BRCA1 haplotypes among healthy women versus breast cancer patients was significantly different; $p=0.02$. Parental consanguinity seems to protect against breast cancer in the Tunisian population
\end{abstract}

Keywords: Consanguinity - breast cancer - BRCA1 - Tunisian patients

Asian Pac J Cancer Prev, 16 (9), 4051-4055

\section{Introduction}

Consanguinity is highly prevalent in many parts of the world. It is defined as a traditional social custom along so many years in different societies. The highest consanguinity rates $(20 \%$ to $50 \%$ of all marriages) are observed in North Africa, the Middle East, Southwest Asia and Southern India (Hamamy and Alwan, 1994). The average inbreeding coefficient reaches 0.02265 in the Saudi population; 0.02442 in Yemen and 0.0157 in Tunisia (Khlat and Khoury, 1991), (Abu-Rabia and Maroun, 2005), (Consortium, 2008). In contrast to these Eastern regions, the mean coefficient of inbreeding was estimated at 0.0002 in the United Kingdom (Smith, 2001). Western European and Northern American countries display low consanguineous marriage rates (less than 1\%) (Liascovich et al., 2001).

The consanguineous offspring has a greater chance to have homozygote alleles by descent. Therefore, the frequency of diseases that are genetically determined may be affected. Studies from Pakistan, Croatia and North America suggest that parental consanguinity increases the risk of breast cancer in women, particularly younger women and those from first-cousin marriages (Grjibovski et al., 2009). However, a study from the Arabian Peninsula reported that consanguinity may lessen the overall risk of breast cancer, especially in younger women (Denic and
Bener, 2001), (Denic et al., 2007).

These contradictory findings create uncertainty about the true risk of breast cancer in women of consanguineous parentage. The involvement of genetic factors along with the role of consanguinity in the development of breast cancer may be different in familial versus sporadic cases. In the present study, we assessed possible effects of parental consanguinity in patients with sporadic and familial breast cancer by comparing their inbreeding level with that in general population. To confirm the population genetics results, we conducted a molecular study on BRCA1 gene homozygous status as potentially indicative of consanguinity. As already described, BRCA1 gene is a suppressor tumor gene, the presence of germ line deleterious mutations lead to high risk of familial breast cancer (Cao et al., 2013). Moreover, several SNPs have been described in this gene. According to previous results several haplotypes of the BRCA1 gene corresponding to the Tunisian population have been established (Cao et al., 2013).

In the present sudy, analysis of nine SNPs; c.442.58delT, c. $2082 \mathrm{C}>\mathrm{T}$, с. $2311 \mathrm{~T}>\mathrm{C}$, c. $2612 \mathrm{C}>\mathrm{T}$, с. $3113 \mathrm{~A}>\mathrm{G}$, c. $3119 \mathrm{G}>\mathrm{A}$, c. $3548 \mathrm{~A}>\mathrm{G}$ c. $4308 \mathrm{~T}>\mathrm{C}$ and $4837 \mathrm{~A}>\mathrm{G}$ allowed to establish the heterozygous and homozygous status of BRCA1 gene across consanguineous and non consanguineous women with breast cancer and healthy controls. 


\section{Materials and Methods}

\section{Study subjects}

For consanguinity study, a total of 155 patients were recruited prospectively from the main cancer hospital (ISA: Salah Azaiez Institute of Carcinology) in Northern Tunisia from 2004 to 2012. All patients were locally born in Tunisia, with histological diagnosis of primary breast cancer. On the basis of family history of the disease, patients were classified into familial (77 cases) and sporadic (78 cases) groups. Familial breast cancer was defined by the presence of more than two cases of breast cancer in the family (Troudi et al., 2007). A group of 23 healthy subjects with no individual or familial cancer history was used as control.

For BRCA1 SNPs analysis, 40 patients with familial breast cancer, 46 patients with sporadic breast cancer were sequenced for the BRCA1 selected nine SNPs along with 34 volunteers Tunisian women randomly selected as healthy controls, varying in age from 24 to 72 years. The peripheral blood was collected into tube and centrifuged at 3,000 rpm for $15 \mathrm{~min}$; the puffy coat was isolated and stored at $-80^{\circ} \mathrm{C}$ until use.

\section{Data collection}

All participants consented to this investigation. All procedures were in agreement with the guidelines for use of human material in research issued by the medical ethics committee of Pasteur Institute of Tunis. An ethical approval was signed by Dr M. Samir BOUBAKER president of the medical ethics committee. Clinical characteristics such as grade and stage, as well as age at diagnosis were ascertained across pathology reports and were taken into account in comparing between sporadic and familial breast cancer to a larger group of "historical controls" (patients recruited at the Salah Azaiez Institute from 2004 to 2012). Clinical and pathological characteristics of sporadic breast cancer cases were comparable to those previously reported in the same population by (Maalej et al., 2008) (Table 1).

\section{Familial and consanguinity analysis}

In all groups, familial pedigrees were established through interviews. Pedigrees were established for each patient or healthy individual for at least three generations. Three factors were taken into account: age at diagnosis of the proband, the familial link between the proband's parents, breast or ovarian cancer cases in the family. The coefficient of inbreeding was calculated for each patient and the mean coefficient of inbreeding $(F)$ was determined for each group in standard manner (Holloway and Sofaer, 1990).

Data were analyzed using a statistical test (Epi Info 7 method) to compare the mean values between two groups. $P$ values less than 0.05 were considered statistically significant.

\section{Direct sequencing}

Genomic DNA was extracted by phenol/Chloroform method from peripheral blood isolated from each sample. Exons of BRCA1 containing the studied SNPs were PCR amplified in a total reaction volume of $50 \mu 1$ including 10 $\mathrm{mM}$ Tris- $\mathrm{HCl}, \mathrm{pH} 8.3,50 \mathrm{mM} \mathrm{KCl}, 1.5-4.5 \mathrm{mM} \mathrm{MgCl}$, $50 \mathrm{mM}$ dNTPs, $10 \mu \mathrm{l}$ of each primer (designed by Centre Jean Perrin; sequences available on demand), $100 \mathrm{ng}$ of genomic DNA and $1 \mathrm{U}$ of either Taq polymerase (Applied Biosystems, Roche). The cycling program of PCR include an initial denaturation at $94^{\circ} \mathrm{C}$ for $5 \mathrm{~min}$, followed by 30 cycles containing $20 \mathrm{~s}$ at of $94^{\circ} \mathrm{C}$. Annealing step at specific temperatures for each primer pair and extension at $72^{\circ} \mathrm{C}$ for $20 \mathrm{~s}$. Amplicons were purified by solid-phase extraction using QIAquick column gel (QIAGEN). The product was sequenced in forward and reverse reactions using Applied Biosystems Taq DyeDeoxy terminator cycle sequencing kit according to the manufacturer's instructions. Cycle sequencing consisted of 25 cycles at $96^{\circ} \mathrm{C}$ for $30 \mathrm{~s}$ and $60^{\circ} \mathrm{C}$ for $30 \mathrm{~s}$. Sequence analysis was performed using SEQMAN (DNAstar, Madison, WI) and SEQSCAPE V2.5 (Applied Biosystems) softwar.

\section{Results}

Cohorts of 155 patients varying in age from 23 to 73 years (median age 48 years) were diagnosed for breast cancer. Among these cases, 78 had sporadic and 77 had familial type of this disease.

We explored inbreeding characteristics of 155 recruited patients with breast cancer; we observe that $87.7 \%$ were not consanguineous, while $12.25 \%$ were inbred (Table 2). This observed rate of consanguinity across patients is lower than that in the analyzed healthy controls which is $34.78 \%$ close to that found in the general population. In general Tunisian population, the mean coefficient of

Table 1. Clinical and Histopathological Characteristics of Patients with Sporadic Breast Cancer Compared to Those Diagnosed in 2004 by (Maalej et al., 2008)

\begin{tabular}{lcc}
\hline Variable & $\begin{array}{c}\text { Sporadic cases } \\
\text { Present study }\end{array}$ & $\begin{array}{c}\text { Historical controls } \\
\text { Patients diagnosed in 2004 (Maalej et al., 2008) }\end{array}$ \\
\hline Number & 78 & 1437 \\
Age at diagnosis & 47 years & 51 years \\
Pathology stage & & \\
$\quad$ Early (0, I, II) & $53.5 \%$ & $59.0 \%$ \\
$\quad$ Late (III, IV) & $46.4 \%$ & $45.9 \%$ \\
$\quad$ Histopathology & $91.3 \%$ & $86.6 \%$ \\
Infiltrating ductal carcinoma & & 0.36 \\
$\quad$ Tumor grade & $40 \%$ & $54.5 \%$ \\
$\quad$ I and II & & 0.14 \\
*P value $<0.05$ is significant & & 0.1
\end{tabular}


Consanguinity Protecting Effect Against Breast Cancer among Tunisian Women: Analysis of BRCA1 Haplotypes

inbreeding previously reported was 0.0157 (Williams et al., 2014). The mean coefficient of inbreeding among breast cancer patients was 0.007 , we did not record any statistical difference of this coefficient between FBC and SBC patients (Table 3). Then we analyzed the frequency of consanguineous and non consanguineous women across breast cancer and healthy control groups. The frequency distribution was significantly different between the two analyzed groups with $\mathrm{P}$ value $=0.001$, and $\mathrm{OR}=0.26$ (0.09-0.7) (Table 4). We also investigated consanguinity according to the age of the disease onset in a group of 136 patients with breast cancer: 89 cases $(65 \%)$ were less than 50 years old at diagnoses, of whom $15.7 \%$ were

Table 2. Inbreeding Characteristics of Breast Cancer Patients

\begin{tabular}{lc}
\hline Inbreeding Characteristics & $\begin{array}{c}\mathrm{N}(\%) \\
\text { Total } \mathrm{N}=155\end{array}$ \\
\hline Mean age & 46.3 years \\
& $(23-73)$ \\
Consanguineous parents & \\
$\quad$ No & $136(87.7 \%)$ \\
$\quad$ Yes & $19(12.25 \%)$ \\
First cousin $(\mathrm{F}=1 / 16)$ & $16(84.2 \%)$ \\
Second cousin $(\mathrm{F}=1 / 64)$ & $1(5.25 \%)$ \\
Double first cousin $(\mathrm{F}=1 / 8)$ & $1(5.25 \%)$ \\
$\mathrm{F}=1 / 13$ & $1(5.25 \%)$ \\
${ }^{*} \mathrm{~N}=$ observed number; $\mathrm{F}=$ coefficient of consanguinity
\end{tabular}

Table 3. Coefficient of Inbreeding in Sporadic and Familial Breast Cancer Patients

\begin{tabular}{lcc}
\hline Patients & $\begin{array}{c}\text { Number } \\
\text { of cases }\end{array}$ & $\begin{array}{c}\text { Mean coefficient } \\
\text { of inbreeding }(\mathrm{F})\end{array}$ \\
\hline Sporadic breast cancer & 78 & 0.008 \\
Familial breast cancer & 77 & 0.007 \\
Total cases & 155 & 0.007 \\
\hline
\end{tabular}

consanguineous. Only $4.25 \%$ of cases aged more than 50 years at diagnoses were consanguineous. This difference is significant with $\mathrm{P}$ value $=0.04$ (Table 5). In order to confirm these results, we undertook a molecular study of BRCA1 gene, investigating homozygous versus heterozygous status. Hence, we analyzed nine SNPs of BRCA1 gene basing on our previous study (Cao et al., 2013), which are; c.442.58delT, c.2082C $>$ T, c.2311T $>$ C, c. $2612 \mathrm{C}>\mathrm{T}$, c. $3113 \mathrm{~A}>\mathrm{G}$, c. $3119 \mathrm{G}>\mathrm{A}$, c. $3548 \mathrm{~A}>\mathrm{G}$ c. $4308 \mathrm{~T}>\mathrm{C}$ and 4837A $>$ G using direct sequencing. Then we established the different haplotypes constituted by the selected nine SNPs using ARLEQUIN 3.0 software and were divided

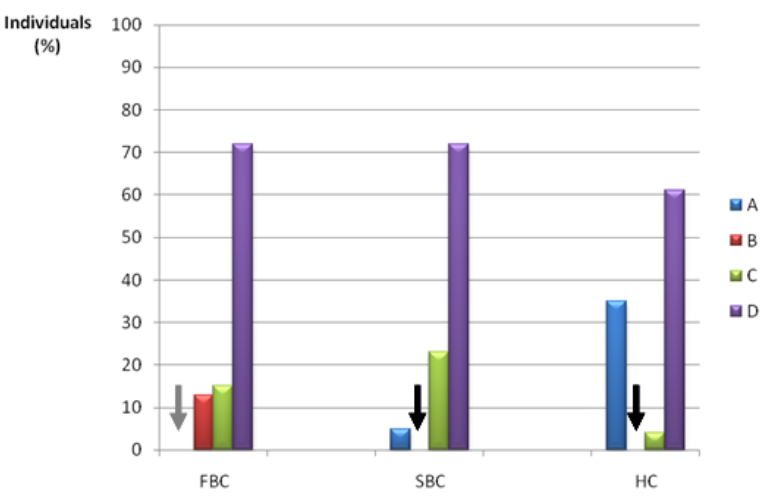

Figure 1. Distribution of Different BRCA1 haplotypes Across Familial and Sporadic Breast Cancer Along with Healthy Controls A: Homozygous BRCA1 haplotypes in consanguineous women, $\mathrm{B}$ : Heterozygous BRCA1 haplotypes in consanguineous women, $\mathrm{C}$ : Homozygous BRCA1 haplotypes in non-consanguineous women, D: Heterozygous BRCA1 haplotypes in non-consanguineous women FBC: Patients with familial breast cancer SBC: Patients with sporadic breast cancer HC: healthy controls Gray arrow: signalizes the total absence of haplotypes A in FBC patients Black arrows: signalize the total absence of haplotypes B in both SBC patients and HC

Table 4. Comparison of Consanguineous and Non-Consanguineous Women's Frequency between Breast Cancer Patients and Healthy Controls

\begin{tabular}{|c|c|c|c|}
\hline & BC Patients $N=155$ & $\begin{array}{l}\text { Healthy controls } \\
\qquad \mathrm{N}=23\end{array}$ & $(\mathrm{P} / \mathrm{OR})$ \\
\hline Consanguineous women & 19 & 8 & $\begin{array}{c}\mathrm{p}=0.001 / \mathrm{OR}=0.26 \\
(0.09-07)\end{array}$ \\
\hline Non-consanguineous women & 136 & 15 & \\
\hline
\end{tabular}

Table 5. Comparison of Consanguinity Rate between Breast Cancer Patients According to the Age at Diagnoses

\begin{tabular}{lccccr}
\hline & Age at diagnoses & Number of patients & Consanguineous patients & Coefficient of inbreeding F & P value \\
\hline Total cases (136) & $<50$ & 89 & $14(15.7 \%)$ & 0.01 & $0.04 *$ \\
& 47 & $2(4.25 \%)$ & 0.0027 & \\
\hline *P value is calculated according to the comparison of \% of consanguineous patients; $\mathrm{P}<0.05$ is significant; $\mathrm{CI}=$ confident interval= $95 \%$
\end{tabular}

Table 6. Comparison of Both Homozygous and Heterozygous BRCA1 Haplotypes Distribution between FBC, SBC and HC

\begin{tabular}{|c|c|c|c|c|c|c|c|}
\hline & FBC40 & SBC46 & $\begin{array}{l}\mathrm{HC} \\
34\end{array}$ & $\begin{array}{c}\mathrm{FBC} v s \mathrm{HC} \\
(\mathrm{P} / \mathrm{OR})\end{array}$ & $\begin{array}{l}\mathrm{SBC} v s \mathrm{HC} \\
(\mathrm{P} / \mathrm{OR})\end{array}$ & $\mathrm{FBC}$ and $\mathrm{SBC} v s \mathrm{HC}(\mathrm{P} / \mathrm{OR})$ & $\begin{array}{c}\mathrm{FBC} v s \mathrm{SBC} \\
(\mathrm{P} / \mathrm{OR})\end{array}$ \\
\hline Homozygous haplotypes & 6 & 13 & 15 & & & & \\
\hline Heterozygous haplotypes & 34 & 33 & 19 & $0.01 / 4.4$ & $0.2 / \mathrm{NS}$ & $0.02 / 2.7$ & $0.2 / \mathrm{NS}$ \\
\hline
\end{tabular}


into homozygous and heterozygous haplotypes. We first analyzed the distribution of homozygous and heterozygous BRCA1 haplotypes among 40 cases of familial breast cancer, 46 cases of sporadic breast cancer and 34 cases of healthy controls (Table 6). We clearly showed significant difference comparing breast cancer patients versus healthy controls with $\mathrm{P}$ value $=0.02$ and $\mathrm{OR}=2.7$ and more particularly in familial breast cancer versus healthy controls with $\mathrm{P}$ value $=0.01$ and $\mathrm{OR}=4.4$ (Table 6$)$. In other words, homozygous genotypes are less frequent in breast cancer patients than in healthy controls. This seems in accordance with the low consanguinity rate observed previously in breast cancer groups.

In order to confirm both previous results, we compared both homozygous and heterozygous BRCA1 haplotypes distribution according to consanguinity in breast cancer patients and healthy controls (Figure 1). Thus, we defined 4 status types shown in figure1. Status (A) corresponds to homozygous BRCA1 haplotypes in consanguineous women, status (B) corresponds to heterozygous BRCA1 haplotypes in consanguineous women, status (C) corresponds to homozygous BRCA1 haplotypes in nonconsanguineous women and status (D) corresponds to heterozygous BRCA1 haplotypes in non- consanguineous women. We first found in the healthy control group, a striking significance of the presence of homozygous BRCA1 haplotypes in consanguineous subjects (A) and of heterozygous BRCA1 haplotypes in non-consanguineous women (D) (Figure1)

In a last step we analyzed the distribution of the homozygous versus heterozygous BRCA1 genotypes according to consanguinity in the three studied groups namely FBC, SBC and HC (Figure1). We observed clearly the absence of the first status (A) in familial breast cancer group and its presence in only $5 \%$ of individuals with sporadic breast cancer while it is present at $35 \%$ among healthy controls suggesting that consanguinity leading to the homozygous BRCA1 genotype protects against breast cancer. Status (B) was absent in healthy controls and in sporadic breast cancer groups, it was only observed among familial breast cancer patients with a frequency of $13 \%$. Status (C) was more observed in breast cancer patients than in healthy controls. Status (D) which is the most frequent is more presented in both familial and sporadic breast cancer than in healthy controls (Figure1).

\section{Discussion}

We studied 155 breast cancer cases recruited from the Salah Azaïez Institute (ISA) of Tunis, the main oncology Center in Northern Tunisia. 1437 breast cancer cases are treated at the ISA annually (Maalej et al., 2008), of which familial cases represent about 5\% (72 cases per year). The 77 analyzed familial cases correspond to the number of familial cases diagnosed per year, suggesting that the sample is representative of familial breast cancer cases in Northern Tunisia. The number of patients with sporadic breast cancer (78) corresponds to around $5 \%$ of such cases in ISA. Their clinical and pathological parameters were similar to those previously published in this population. Therefore, this group is representative of sporadic breast cancer in Tunisian population.

Overall, patients with breast cancer have lower rates of both consanguineous marriages and mean coefficient of inbreeding than these in general population of Tunis. These results suggest the protective effect assigned to consanguinity against breast cancer, since we observed an excess of consanguineous women in healthy controls comparing to consanguineous women in breast cancer patients. This finding is similar to that of Denic et al., 2001, who demonstrated that in some areas, parental consanguinity was more frequent among healthy women than among those with breast cancer (Denic and Bener, 2001). More recently similar results were obtained in Moroccan population (Elalaoui et al., 2013).

Moreover, we analyzed in this study consanguinity according to age of breast cancer onset. Patients older than 50 years at diagnoses had a coefficient of inbreeding lower than that of patients younger than 50 years at diagnoses, independently of the sporadic or familial presentation of the disease with $\mathrm{P}$ value $=0.04$. For patients younger than 50 years old at diagnoses, the average coefficient of inbreeding was similar to that of the general population, suggesting that consanguinity would be protective against breast cancer after 50 years old. In contrast, Middle Eastern women whose parents are consanguineous present a lower risk of developing breast cancer before age 50 than women of non-consanguineous parents. This effect was not found with those older than 50 years old at diagnoses (Denic and Bener, 2001) (Moore et al., 2014).

To further confirm these results, we performed analysis of BRCA1 haplotypes taking into account nine SNPs already described in our Tunisian population by Troudi et al., 2008. Globally we expected to find more homozygous haplotypes in women from consanguineous marriages than from non-consanguineous ones. Interestingly, such a status was not found in consanguineous patients with familial breast cancer. In fact, in FBC patients, not only consanguinity rate is low, but even in cases of consanguineous marriages, none of those patients is homozygous at the BRCA1 gene. This observation might be indicative of a selection against the BRCA1 homozygous status in FBC, and is likely related to the deleterious mutations of BRCA1 gene that was described as the major cause of familial breast cancer onset (Moore, 2013). These mutations are expected to be lethal at homozygous state. Indeed, several studies showed the lethal effect in embryos mice, of BRCA1 knockout homozygous genotype (Hohenstein et al., 2001). The outcomes of homozygous deleterious mutations of this gene in humans are still unknown. But we can suggest that in consanguineous families with deleterious BRCA1 mutations, three genotypes are possible: the first; without mutations gives healthy subjects, the second; with deleterious mutations at heterozygous state leads to the breast cancer and the third; with deleterious mutation at homozygous state which is lethal. In our study, absence of parental consanguinity in all six patients with BRCA1 deleterious mutations supports this hypothesis. On the contrary, all consanguineous healthy controls appeared homozygous at BRCA1 gene. In these cases, homozygous BRCA1 haplotypes are in fact too much frequent 
Consanguinity Protecting Effect Against Breast Cancer among Tunisian Women: Analysis of BRCA1 Haplotypes

comparatively to that expected from consanguineous cases. This observation could be related to a selection in favor of the BRCA1 homozygous status and could be explained by the presence of BRCA1 alleles that do not carry any deleterious mutation and that are protective against breast cancer mostly at homozygous state. The functional significance of this protective effect needs to be more investigated. However, we cannot exclude that the homozygous BRCA1 genotype was over evaluated in this study, since other SNPs in BRCA1 gene do exist and were not analyzed.

In conclusion, parental consanguinity seems to protect against breast cancer in Tunisian population especially for those older than 50 years. Further confirmation of these findings and study of mechanism by which inbreeding lowers odds of malignancy deserve additional studies.

\section{Acknowledgements}

The authors would like to thank all persons contributing to the realization of this work in Salah Azaiz Institute of Tunis, Laboratory of genetic immunology and human pathology. It was supported by IncoMed project Number: ICA-CT-2002-10005

\section{References}

Abu-Rabia S, Maroun L (2005). The effect of consanguineous marriage on reading disability in the Arab community. Dyslexia, 11, 1-21.

Cao W, Wang X, Gao Y, et al (2013). BRCA1 germ-line mutations and tumor characteristics in eastern Chinese women with familial breast cancer. Anat Rec, 296, 273-8.

Consortium AU-ACR (2008). Cancer registration literature update (2006-2008). Asian Pac J Cancer Prev, 9, 165-82.

Denic S, Bener A (2001). Consanguinity decreases risk of breast cancer--cervical cancer unaffected. Br J Cancer, 85, 1675-9.

Denic S, Frampton C, Nicholls MG (2007). Risk of cancer in an inbred population. Cancer Detect Prev, 31, 263-9.

Elalaoui SC, Jaouad IC, Laarabi FZ, et al (2013). Low level of consanguinity in moroccan families at high risk of breast cancer. Asian Pac J Cancer Prev, 14, 723-6.

Grjibovski AM, Magnus P, Stoltenberg C (2009). Decrease in consanguinity among parents of children born in Norway to women of Pakistani origin: a registry-based study. Scand J Public Health, 37, 232-8.

Hamamy H, Alwan A (1994). Hereditary disorders in the Eastern Mediterranean Region. Bull WHO, 72, 145-54.

Hohenstein P, Kielman MF, Breukel C, et al (2001). A targeted mouse Brca1 mutation removing the last BRCT repeat results in apoptosis and embryonic lethality at the headfold stage. Oncogene, 20, 2544-50.

Holloway SM, Sofaer JA (1990). Coefficients of relationship by isonymy among the parents of Scottish twins: a test of familial aggregation. Hum Biol, 62, 429-35.

Khlat M, Khoury M (1991). Inbreeding and diseases: demographic, genetic, and epidemiologic perspectives. Epidemiol Rev, 13, 28-41.

Liascovich R, Rittler M, Castilla EE (2001). Consanguinity in South America: demographic aspects. Hum Hered, 51, 27-34.

Maalej M, Hentati D, Messai T, et al (2008). Breast cancer in Tunisia in 2004: a comparative clinical and epidemiological study. Bull Cancer, 95, 5-9.

Moore MA (2013). Overview of cancer registration research in the Asian Pacific from 2008-2013. Asian Pac J Cancer Prev, 14, 4461-84.

Moore MA, Sangrajrang S, Bray F (2014). Asian Cancer Registry Forum 2014 - regional cooperation for cancer registration: priorities and challenges. Asian Pac J Cancer Prev, 15, 1891-4.

Smith MT (2001). Estimates of cousin marriage and mean inbreeding in the United Kingdom from 'birth briefs'. $J$ Biosoc Sci, 33, 55-66.

Troudi W, Uhrhammer N, Sibille C, et al (2007). Contribution of the BRCA1 and BRCA2 mutations to breast cancer in Tunisia. J Hum Genet, 52, 915-20.

Williams CK, Cristina Stefan D, Rawlinson F, et al (2014). The African Organisation for Research and Training in Cancer and its conferences: a historical perspective and highlights of the Ninth International Conference, Durban, South Africa, 21-24 November 2013. Ecancermedicalscience, 8, 396. 